\title{
Quenching Star Formation in the Green Valley: The Mass Flux at Intermediate Redshifts
}

\author{
Thiago S. Gonçalves ${ }^{1} \&$ D. Christopher Martin ${ }^{1}$ \\ ${ }^{1}$ California Institute of Technology, \\ 1200 E. California Blvd. MC 278-17, Pasadena CA 91107, USA \\ email: tsg@astro.caltech.edu
}

\begin{abstract}
We have obtained several hundred very deep spectra with DEIMOS/Keck in order to estimate the galactic mass flux density at intermediate redshifts $(0.6<z<0.9)$ from the "blue cloud" to the red sequence across the so-called "green valley", the intermediate region in the color-magnitude plot between those two populations. We use spectral indices (specifically $\mathrm{D}_{n}(4000)$ and $\left.\mathrm{H}_{\delta, A}\right)$ to determine star formation histories. Together with an independent measurement of number density of galaxies in each bin of the color-magnitude plot, one can infer the rate at which galaxies from a given sample are transiting through that bin. Measuring this value for all magnitude values, studies at lower redshift determined that the mass flux density in the green valley is comparable to both the mass build-up rate of the red sequence and the mass loss rate from the blue cloud. We show preliminary results for our intermediate redshift sample.
\end{abstract}

Keywords. galaxies: evolution - galaxies: general

\section{Introduction}

It has been shown that galaxies in the low-redshift universe present a bimodal distribution, with a clear distinction between blue, star-forming spirals and red ellipticals with an older stellar population (e.g. Balogh et al. 2004, Baldry et al. 2004). More recently, it has been discovered that the bimodality is already in place at redshift $\mathrm{z} \sim 1$, when the universe was approximately half its present age (Willmer et al. 2006). Although there is wide agreement that most galaxies are divided into two very distinct groups, we do not yet understand the physical processes responsible for transforming galaxies. Due to the low number of objects with intermediate colors, we are led to believe the migration occurs quickly, but what exactly are the properties of the so-called green valley galaxies? (Fig. 1)

\section{Mass Flux from Blue to Red}

One important aspect of star formation quenching is how fast the transition is occurring. With that in mind, we employ a simplified model to measure quenching timescales of green valley galaxies. Simply put, the idea is to measure spectral indices - more specifically, $\mathrm{D}_{n}(4000)$ and $\mathrm{H}_{\delta, A}$ - to infer star formation histories for individual galaxies. Kauffmann et al. (2003) have shown that the $4000 \AA$ break and the $\mathrm{H}_{\delta}$ absorption lines are a good proxy for distinct star formation histories. As the stellar population in a given galaxy grows old and the $4000 \AA$ becomes more prominent, continuous or bursty star formation episodes will yield different tracks across the $\mathrm{D}_{n}(4000)$ vs. $\mathrm{H}_{\delta, A}$ plane (Martin et al. 2007). 
By using a simple model of continuous star formation rate followed by an exponential decline, one can predict the spectral indices at a given time. Further comparison with actual measurements, assuming the validity of such simple model, will help determine the timescale of the exponential decline for a given single object. If in addition we have information of mass density for a given color-magnitude diagram (CMD) bin, the data combined can yield the total mass flux across the green valley. (Fig. 2)

Wyder et al. (2007) have found that the green valley is better determined by a combination of ultraviolet and optical colors. Unlike for optical colors alone, NUV $-\mathrm{r}$ diagrams show a dynamic range of approximately six magnitudes, with very clear separation between the blue and the red sequences. This is ideal to select galaxies with intermediate colors.

A cross-match between the SDSS catalog and GALEX Medium Imaging Survey (MIS) allows for a wealth of information on galaxies at low redshift, $0.1<z<0.3$. By analyzing that data and extracting the aforementioned spectral indices, Martin et al. (2007) have been able to determine the mass flux through the green valley in the low-redshift universe, and have come to the conclusion that this number is comparable both to the mass loss from the blue sequence and the build-up of the red sequence (Faber et al. 2007; Bell et al. 2003).

\section{The Mass Flux at Intermediate Redshifts}

Galaxy evolution studies have shown that processes occur differently at different ages of the universe. Star forming galaxies in the present day are less massive and present smaller star formation rates than those at high redshift, when the universe was younger, in what has been described as downsizing (Cowie et al. 1996). One might expect that, likewise, the mass flux through the green valley will vary according to redshift.

To test that hypothesis, we have obtained several spectra of green valley galaxies at intermediate redshifts, $0.55<z<0.9$. These galaxies were selected by rest frame NUV $-\mathrm{r}$

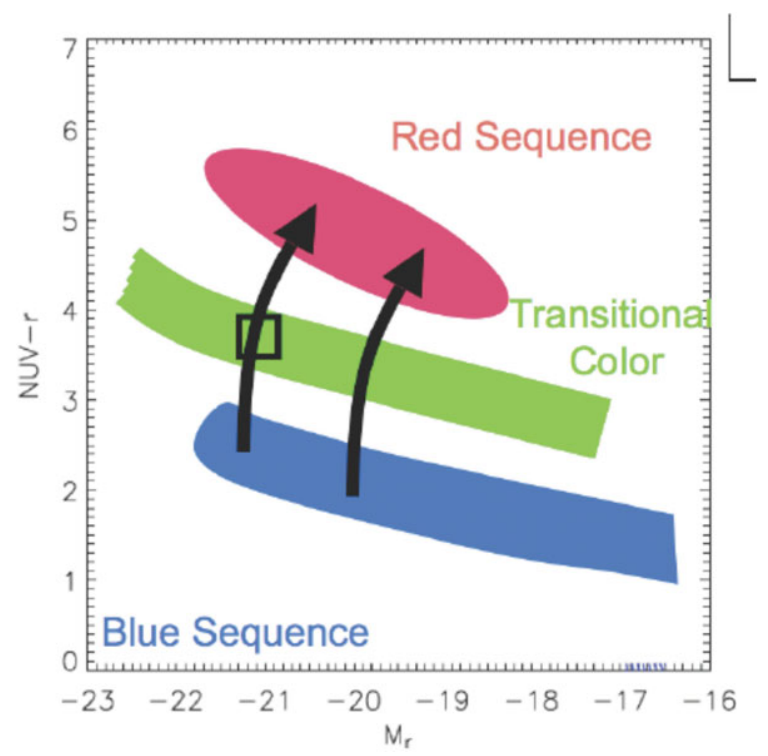

Figure 1. Schematic view of green valley galaxies in the color-magnitude diagram (CMD), with $r$ magnitude in the $\mathrm{x}$-axis and NUV-r colors in the y-axis. 
colors, in the same manner as for the low-z sample, with $3<\mathrm{NUV}-\mathrm{r}<4.5$. We have shown that the CMD is remarkably similar at higher redshifts, with the red and blue sequences occupying comparable loci in the diagram.

The challenge in this case is to be able to measure equivalent widths of low-luminosity $(\mathrm{r} \sim 24 \mathrm{mag})$ galaxies. Stacked spectra might yield some results, but galaxy properties within a given CMD bin vary widly, yielding large uncertainties. In addition, the possibility of determining quenching timescales for a single galaxy allows for a direct comparison with other galactic properties, such as morphologies and AGN activity. We have therefore obtained very deep spectra ( $\sim 8 \mathrm{hr}$ in a $10 \mathrm{~m}$-class telescope) of green valley galaxies at intermediate redshifts.

The DEIMOS instrument at the Keck telescope has a wide enough field of view $(\sim 15$ arcmin) that we have a sufficient number of green valley objects in a given mask. By observing a mask per night, we have obtained spectra of approximately 200 objects, with enough signal-to-noise to be able to reliably measure $\mathrm{H}_{\delta, A}$ for all of them. The results are shown in Fig. 3. We conclude that the models reliably predict the observed values, although the data scatter at this point does not yet allow for a determination of timescales for these objects.

We are in the process of cross-correlating our results with AGN activity, taking emission line diagnostics and X-ray emissivity as proxies. AGN have been shown to reside preferentially in the green valley, and are believed to play an important role in the quenching of star formation (Martin et al. 2007; Nandra et al. 2007). If that is true, we would expect stronger AGN to present faster quenching timescales.

It is still unclear what processes at any redshift drive galaxies across the CMD to become passively evolving ellipticals. The combined photometric NUV $-\mathrm{r}$ selection of green valley galaxies and subsequent detailed, deep spectral analysis of individual objects provide the ideal tools to study this transitioning population, allowing us to address one of the most fundamental, open questions in galaxy evolution.

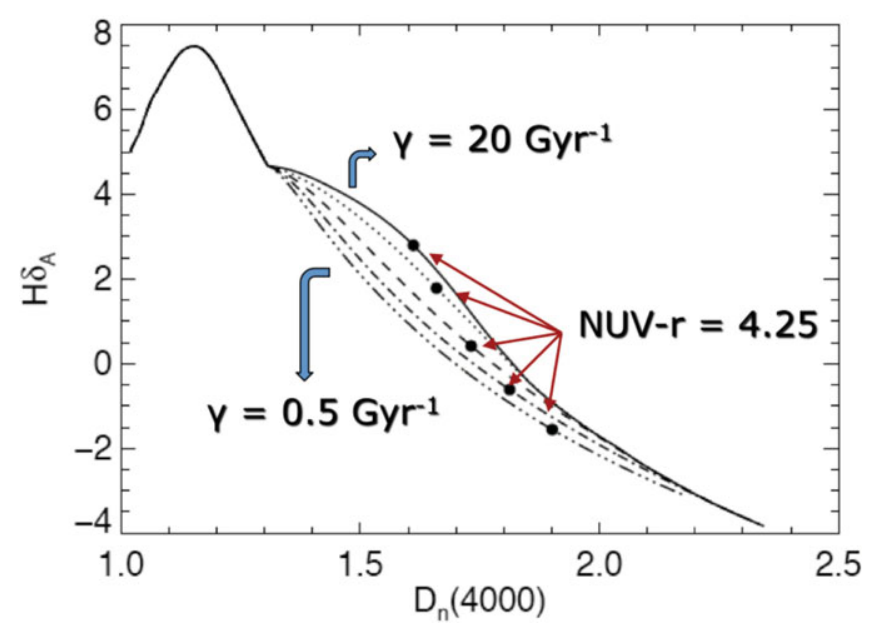

Figure 2. Diagram displaying distinct tracks across the $\mathrm{D}_{n}(4000)$ vs. $\mathrm{H}_{\delta, A}$ plane. Galaxies with fast quenching timescales (solid line) are clearly distinguishable from slowly quenching ones (dot-dashed lines). By overplotting actual spectral measurements, one can infer quenching timescales of individual green valley galaxies. Galaxy models with NUV $-\mathrm{r}=4.25$ colors are highlighted as solid circles. 


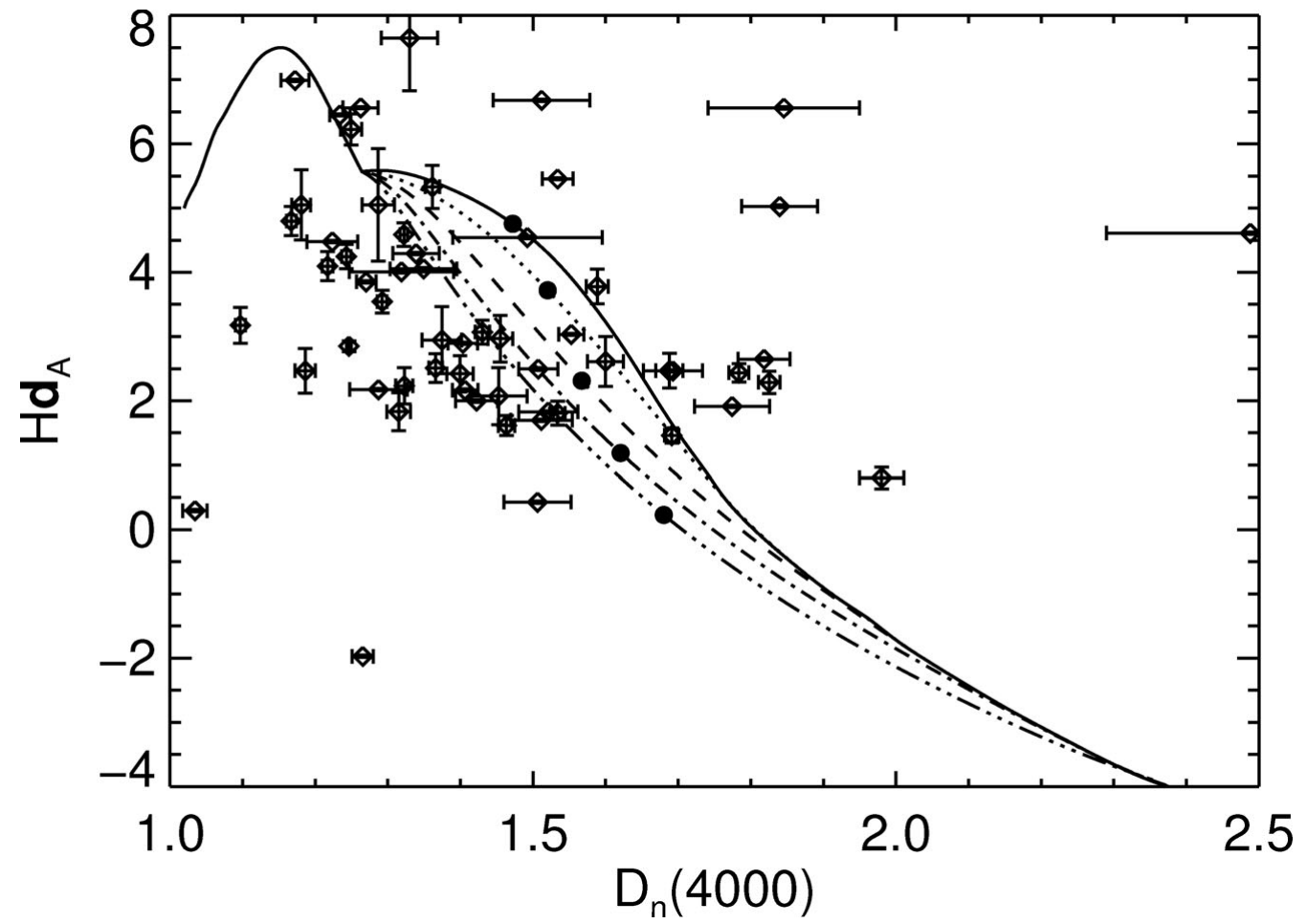

Figure 3. Example of real data at redshifts $0.55<z<0.9$. Most galaxies are bluer than $\mathrm{NUV}-\mathrm{r}=4.25$, and therefore are left of the solid circles, as expected. These are among the deepest spectra ever taken for a large sample of galaxies at such redshifts, and the intrinsic errors for absorption line measurements of $\mathrm{r} \sim 24$ galaxies are remarkably small. We expect a more careful data reduction will dramatically reduce systematic errors. (Gonçalves \& Martin 2010)

\section{References}

Baldry, I. K., Balogh, M. L., Bower, R., Galzebrook, K., \& Nichol, R. C. 2004, ApJ, 600,681

Balogh, M. L., Baldry, I. K., Nichol, R. C., Miller, C. Bower, R., \& Galzebrook, K. 2004, ApJ (Letters), 615, 101

Bell, E. F., McIntosh, D. H., Katz, N., \& Weinberg, M. D. 2003, ApJ (Supplement Series), 149, 289

Cowie, L. L., Songaila, A., Hu, E. M., \& Cohen, J. G. 1996, AJ, 112, 839

Faber, S. M., Willmer, C. N. A., Wolf, C. et al. 2007, ApJ, 665, 265

Gonçalves, T. S. \& Martin, D. C. 2010, in prep.

Kauffmann, G., Heckman, T. M., White, S. D. M. et al. 2003, MNRAS, 341, 33

Martin, D. C., Wyder, T. K., Schiminovich, D. et al. 2007, ApJ (Supplement Series), 173, 342

Nandra, K., Georgakakis, A., Willmer, C. N. A. et al. 2007, ApJ (Letters), 660, 11

Willmer, C. N. A., Faber, S. M., Koo, D. C. et al. 2006, ApJ, 647, 853

Wyder, T. K., Martin, D. C., Schiminovich, D. et al. 2007, ApJ (Supplement Series), 173, 293 